\title{
Le rémifentanil est une alternative efficace au propofol pour l'analgésie auto-contrôlée en endoscopie digestive
}

\author{
[Remifentanil is an effective alternative to propofol for patient-controlled analge- \\ sia during digestive endoscopic procedures]
}

Lionel Bouvet MD, Bernard Allaouchiche MD PhD, Frédéric Duflo MD, Richard Debon MD, Dominique Chassard MD PhD, Emmanuel Boselli MD

\begin{abstract}
Objectif : A la différence du propofol, l'auto-administration de rémifentanil en sédation pour endoscopie digestive n'a jamais été évaluée dans la littérature. Nous avons voulu comparer l'efficacité et la tolérance de l'auto-administration de rémifentanil ou de propofol par le patient lors d'endoscopies digestives.
\end{abstract}

Méthode : L'étude prospective, randomisée en simple aveugle, a été réalisée auprès de 77 patients devant bénéficier d'endoscopie digestive, répartis en deux groupes : groupe $\mathrm{R}=$ administration de rémifentanil (débit continu $5 \mu \mathrm{g} \cdot \mathrm{kg}^{-1} \cdot \mathrm{h}^{-1}$, bolus $25 \mu \mathrm{g}$, période réfractaire cinq minutes) et groupe $\mathrm{P}=$ administration de propofol (débit continu $2 \mathrm{mg} \cdot \mathrm{kg}^{-1} \cdot \mathrm{h}^{-1}$, bolus $0,5 \mathrm{mg} \cdot \mathrm{kg}^{-1}$, période réfractaire dix minutes). Un bolus de I $\mathrm{mg} \cdot \mathrm{kg}^{-1}$ de propofol était administré avant le début de l'examen en cas d'anxiété importante. Des boli supplémentaires de $25 \mu \mathrm{g}$ de rémifentanil ou de $0,5 \mathrm{mg} \cdot \mathrm{kg}^{-1}$ de propofol étaient administrés en cas de plainte survenant en période réfractaire. L'évaluation a porté sur l'efficacité analgésique pendant l'examen et sur la satisfaction des patients. Le niveau de sédation pendant l'examen et la survenue d'effets indésirables ont été analysés.

Résultats : La satisfaction des patients était élevée et comparable dans les deux groupes, avec un nombre de patient réveillés et orientés significativement plus élevé dans le groupe R. La tolérance hémodynamique et respiratoire était comparable dans les deux groupes, malgré la survenue de deux épisodes de désaturation dans le groupe $R$. L'incidence des nausées était significativement plus importante dans le groupe $R$.

Conclusions : L'auto-administration de rémifentanil en sédation pour endoscopie digestive est aussi efficace que l'auto-administration de propofol et peut être proposée notamment lorsque la perte de connaissance du patient n'est pas souhaitée.
Purpose: Unlike propofol, the self-administration of remifentanil for sedation in gastrointestinal endoscopies has never been evaluated formally. We wanted to compare the efficacy and tolerance of patient self-administration of remifentanil vs propofol during gastrointestinal endoscopy.

Method: This prospective randomized, single-blinded study, included 77 patients undergoing gastrointestinal endoscopy. Patients were divided into two groups: group $R$ received remifentanil $\left(5 \mu \mathrm{g} \cdot \mathrm{kg}^{-1} \cdot \mathrm{hr}^{-1}\right.$ infusion, $25 \mu \mathrm{g}$ boli, refractory period of five minutes) and group $P$ received propofol (2 $\mathrm{mg} \cdot \mathrm{kg}^{-1} \cdot \mathrm{hr}^{-1}$ infusion, $0.5 \mathrm{mg} \cdot \mathrm{kg}^{-1}$ boli, refractory period of ten minutes). A I mg. $\mathrm{kg}^{-1}$ bolus of propofol was administered before the procedure began in cases of marked anxiety. Additional boli of $25 \mu \mathrm{g}$ of remifentanil or $0.5 \mathrm{mg} \cdot \mathrm{kg}^{-1}$ of propofol were administered when patients complained during the refractory period. The evaluation targeted analgesic efficacy during the procedure and patient satisfaction. The degree of sedation during the procedure and the occurrence of adverse reactions were analyzed.

Results: Patient satisfaction was high and comparable in both groups, with the number of awake and oriented patients being significantly higher in group $R$. Hemodynamic and respiratory tolerance was comparable in both groups, despite two episodes of desaturation in group $R$. The incidence of nausea was significantly higher in group $R$.

Conclusions: The self-administration of remifentanil for sedation during gastrointestinal endoscopies is as effective as the self-administration of propofol and can be offered to patients, especially when it is desirable that they remain conscious during the procedure.

Du Service d'Anesthésie-Réanimation, Hôtel-Dieu, Lyon, France.

Adresser la correspondance à : Dr Emmanuel Boselli, Service d'Anesthésie-Réanimation, Hôtel-Dieu, 1 place de l'hôpital, 69288 Lyon cedex 02, France. Téléphone : +33 472413170 ; Télécopieur : +33472413135; Courriel : emmanuel.boselli@chu-lyon.fr

Accepted for publication May 5, 2003.

Revision accepted November 14, 2003. 
$\mathrm{L}$ ES endoscopies digestives sont des examens désagréables et parfois douloureux, justifiant l'administration d'agents sédatifs afin d'assurer le confort et la coopération du patient. Le propofol a été évalué avec succès en sédation contrôlée par le patient (SCP) pour endoscopie digestive. ${ }^{1,2}$ Cependant, la perte de connaissance et parfois l'amnésie procurées par les agents hypnotiques, dont le propofol, ne semblent pas être indispensables à l'efficacité de la sédation. ${ }^{3,4}$

Le rémifentanil est un morphinomimétique qui a déjà été proposé en perfusion continue lors de colonoscopies en ventilation spontanée, et en SCP lors de lithotrities extracorporelles et en analgésie obstétricale. ${ }^{5-7}$ L'auto-administration de rémifentanil en endoscopie digestive n'a pas été évaluée à ce jour.

L'objectif de cette étude était de comparer l'efficacité et la tolérance de la SCP utilisant du rémifentanil ou du propofol lors d'endoscopies digestives.

\section{Patients et méthodes}

Après accord du Comité d'Éthique de l'hôpital et recueil du consentement éclairé, 77 patients âgés de plus de 18 ans de classe ASA I ou II, devant bénéficier d'endoscopie digestive haute et/ou basse programmée non-interventionnelle, ont été inclus dans cette étude prospective, randomisée en simple aveugle.

L'anxiété des patients était évaluée avant l'examen. En cas d'anxiété importante, un bolus de $1 \mathrm{mg} \cdot \mathrm{kg}^{-1} \mathrm{de}$ propofol était administré cinq minutes avant le début de l'examen. De l'oxygène était administré par voie nasale à un débit de $3 \mathrm{~L} \cdot \mathrm{min}^{-1}$ à tous les patients.

Les patients ont été randomisés en deux groupes : dans le groupe P, la SCP associait la perfusion de propofol (débit : $2 \mathrm{mg} \cdot \mathrm{kg}^{-1} \cdot \mathrm{h}^{-1}$ ), à l'auto-administration de boli supplémentaires de $0.5 \mathrm{mg} \cdot \mathrm{kg}^{-1}$, suivant une période réfractaire de dix minutes. Dans le groupe $\mathrm{R}$, la sédation associait la perfusion de rémifentanil (débit : $5 \mu \mathrm{g} \cdot \mathrm{kg}^{-1} \cdot \mathrm{h}^{-1}$ ) à l'auto-administration de boli supplémentaires de $25 \mu \mathrm{g}$, suivant une période réfractaire de cinq minutes. Dans les deux groupes, la perfusion continue était débutée cinq minutes avant le début de l'endoscopie. Une pompe de SCP programmable (IVAC $®$ PCAM ${ }^{\circledR}$, Alaris Medical Systems Inc., San Diego, CA, USA) était utilisée. En cas de plainte survenant en période réfractaire, $25 \mu \mathrm{g}$ de rémifentanil (groupe R) ou $0.5 \mathrm{mg} \cdot \mathrm{kg}^{-1}$ de propofol (groupe P) étaient administrés et éventuellement renouvelés par l'anesthésiologiste.

L'évaluation a porté sur l'intensité de la douleur ressentie pendant l'examen, à l'aide d'une échelle visuelle analogique (EVA) graduée de 0 (pas de douleur) à $10 \mathrm{~cm}$ (douleur maximale) toutes les cinq minutes, et sur la satisfaction des patients recueillie lors du départ de la salle de surveillance post-interventionnelle (SSPI). Le niveau de sédation ( 1 : patient réveillé et orienté ; 2 : patient somnolent, répondant à la commande verbale ; 3 : patient endormi, réagissant à la stimulation tactile; 4 : patient non réveillable), ${ }^{8}$ la tolérance hémodynamique (fréquence cardiaque et pression artérielle systolique et diastolique relevées toutes les cinq minutes) et respiratoire (fréquence respiratoire relevée toutes les cinq minutes ; enregistrement des épisodes de désaturation définis par $\mathrm{SpO}_{2}<92 \%$ pendant au moins une minute), la survenue de nausées ou de vomissements et la durée de séjour en SSPI ont également été analysés.

L'analyse statistique a fait appel à l'analyse de variance à mesures répétées pour les variables continues ou au test de Fisher pour les variables nominales (logiciel StatView ${ }^{\mathrm{TM}}$ 5.0, SAS Inst., Cary, NC, USA). La significativité des tests a été retenue pour $P<0.05$.

TABLEAU I Caractéristiques démographiques et endoscopiques

\begin{tabular}{lll}
\hline & Groupe $R(n=41)$ & Groupe P $(n=36)$ \\
\hline Age (ans) & $61 \pm 15$ & $59 \pm 14$ \\
Poids (kg) & $68 \pm 15$ & $67 \pm 10$ \\
Ratio homme/femme $n$ & $19 / 22$ & $17 / 19$ \\
ASA I $n$ (\%) & $13(32)$ & $8(22)$ \\
ASA II $n(\%)$ & $28(68)$ & $28(78)$ \\
Score d'anxiété $n$ (\%) & & \\
$\quad 1$ : aucune, patient calme & $27(66)$ & $20(56)$ \\
$\quad$ : modérée & $12(29)$ & $12(33)$ \\
OGD $n$ intense, patient peu coopérant & $2(5)$ & $4(11)$ \\
Colonoscopie $n$ (\%) & $21(51)$ & $12(33)$ \\
OGD et colonoscopie $n$ (\%) & $2(5)$ & $5(14)$ \\
Durée (min) & $18(44)$ & $19(53)$ \\
\hline
\end{tabular}

OGD = oeso-gastro-duodénoscopie; Résultats exprimés en moyenne \pm DS ou n (\%). 
TABLEAU II Satisfaction et tolérance

\begin{tabular}{|c|c|c|}
\hline & Groupe $R(n=41)$ & Groupe $P(n=36)$ \\
\hline \multicolumn{3}{|l|}{ Satisfaction $n(\%)$} \\
\hline très satisfaits & $24(58)$ & $18(50)$ \\
\hline satisfaits & $14(34)$ & $17(47)$ \\
\hline peu satisfaits & $3(7)$ & $1(3)$ \\
\hline non satisfaits & $0(0)$ & $0(0)$ \\
\hline Désaturation $\left(\mathrm{SpO}_{2}<92 \%\right) n(\%)$ & $2(5)$ & $0(0)$ \\
\hline Score de sédation $>1 n(\%)$ & $22(54)$ & $31(86)^{*}$ \\
\hline Nausées $n(\%)$ & $12(29)$ & $0(0) * *$ \\
\hline Vomissements $n(\%)$ & $2(5)$ & $0(0)$ \\
\hline Durée de séjour en SSPI (min) & $37 \pm 13$ & $33 \pm 7$ \\
\hline
\end{tabular}

Résultats exprimés en moyenne \pm DS ou $n(\%)$. SSPI $=$ salle de surveillance post-interventionnelle.

${ }^{*} P<0.05 ; * * P<0.001$.

\section{Résultats}

Soixante-dix-sept patients âgés de 23 à 89 ans ont été inclus dans l'étude. Les caractéristiques démographiques et endoscopiques des deux groupes étaient comparables (Tableau I).

La satisfaction des patients était élevée et comparable dans les deux groupes (Tableau II). Les scores d'EVA étaient comparables et restaient inférieurs à $3 \mathrm{~cm}$ (Figure 1). Le nombre de patients réveillés et orientés pendant l'examen était significativement plus élevé dans le groupe $\mathrm{R}$ (Tableau II).

La tolérance hémodynamique et respiratoire était comparable dans les deux groupes (Figure 2). Deux épisodes de désaturation ont été observés dans le groupe $\mathrm{R}$, dont une apnée ayant nécessité la ventilation manuelle du patient pendant moins de deux minutes (Tableau II).

La survenue de nausées était significativement plus importante dans le groupe R. Seuls deux épisodes de vomissements sont survenus dans le groupe $\mathrm{R}$, durant le séjour des patients en SSPI (Tableau II).

\section{Discussion}

La SCP utilisant du propofol a été évaluée lors de lithotrities extracorporelles, en complément d'anesthésies régionales, ainsi qu'en endoscopie digestive, utilisant du propofol administré seul ou associé à l'auto-administration d'alfentanil. ${ }^{1,2,8,9}$ La SCP est une technique anesthésique efficace, notamment pour des actes peu douloureux où elle permet d'obtenir une sédation adaptée à chaque patient en limitant les risques de surdosage. ${ }^{1,2,8,9}$

Aucune étude n'avait évalué l'auto-administration du rémifentanil en endoscopie digestive. L'autoadministration de rémifentanil a déjà été proposée lors de lithotrities extracorporelles et en analgésie obstétri-

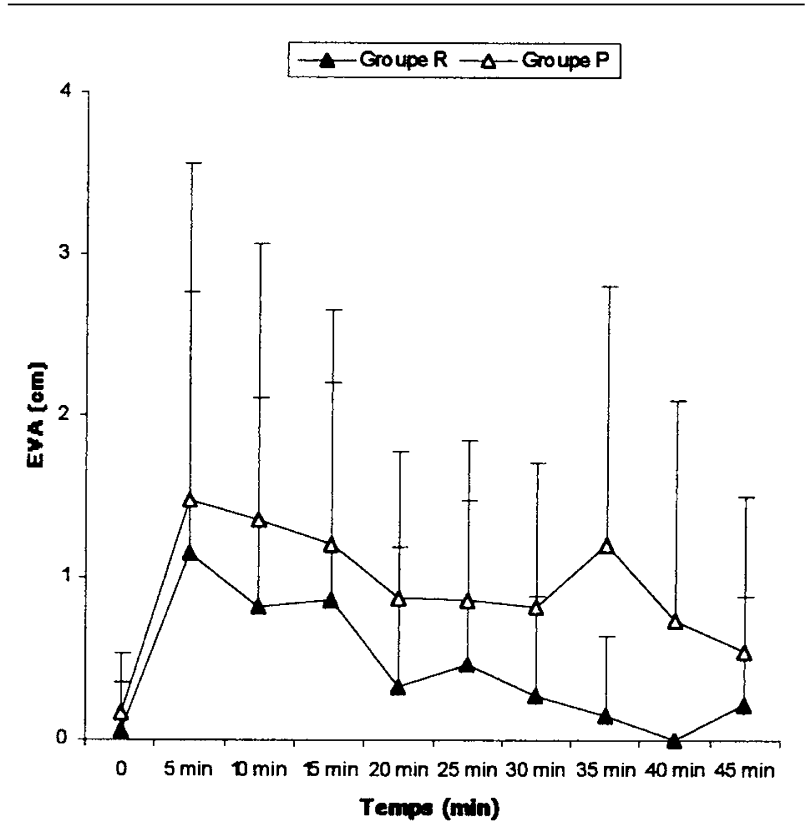

$\overline{\text { FIGURE } 1 \text { Évolution des scores de douleur (EVA) pendant l'ex- }}$ amen endoscopique. Les barres d'erreur indiquent les déviations standard.

cale. ${ }^{6,7}$ Notre étude suggère que la SCP utilisant du rémifentanil en endoscopie digestive est aussi efficace que l'auto-administration de propofol, avec une sédation moindre. L'effet sédatif des agents hypnotiques ne semble pas augmenter le confort des patients lors d'endoscopies digestives ou d'actes peu douloureux. ${ }^{3,4,10}$ L'absence d'effet anxiolytique du rémifentanil a néanmoins nécessité l'administration 


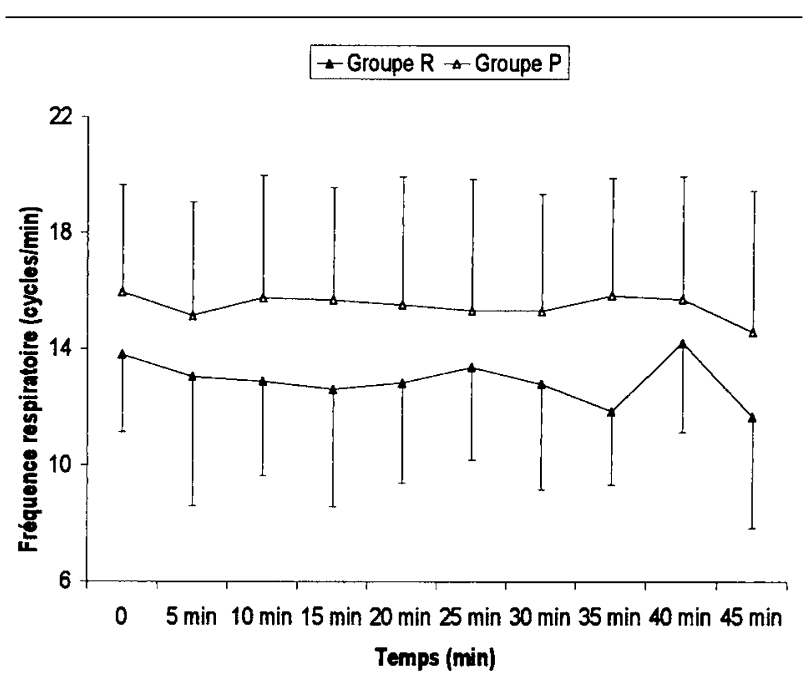

FIGURE 2 Évolution de la fréquence respiratoire pendant l'examen endoscopique. Les barres d'erreur indiquent les déviations standard.

d'un bolus de propofol cinq minutes avant le début de l'examen chez deux patients du groupe R (Tableau I).

Les deux épisodes de désaturation survenus dans le groupe $\mathrm{R}$ ont eu lieu lors de colonoscopies. L'hypoxémie, lors des colonoscopies, est le plus souvent en rapport avec une sédation excessive, s'accompagnant d'une dépression respiratoire. ${ }^{3}$ Notre protocole propose une perfusion continue de rémifentanil supérieure à celle proposée dans d'autres études évaluant l'auto-administration de rémifentanil en analgésie obstétricale ou la perfusion continue de rémifentanil en sédation pour colonoscopies. ${ }^{5,7}$ Ainsi, une diminution, voire la suppression, de la perfusion continue probablement excessive de rémifentanil dans notre protocole devrait être évaluée afin de réduire le risque de dépression respiratoire.

Les nausées-vomissements postopératoires font partie des effets secondaires dose-dépendants du rémifentanil. ${ }^{6,10}$ Ils peuvent compromettre le confort des patients et retarder l'aptitude à la rue. La diminution de la perfusion continue de rémifentanil permettrait probablement de réduire leur incidence.

En conclusion, la SCP utilisant du rémifentanil ou du propofol est une technique efficace et appréciée des patients. L'utilisation du rémifentanil semble intéressante lorsque la perte du contact verbal avec le patient n'est pas souhaitée par l'endoscopiste. La SCP reste néanmoins un acte anesthésique qui nécessite une surveillance médicale continue. D'autres études utilisant le rémifentanil restent à réaliser afin de proposer une SCP plus sûre en endoscopie digestive, notamment en évaluant une SCP sans débit continu.

\section{Références}

1 Roseveare C, Seavell C, Patel P, et al. Patient-controlled sedation and analgesia, using propofol and alfentanil, during colonoscopy: a prospective randomized controlled trial. Endoscopy 1998; 30: 768-73.

2 Heiman DR, Tolliver BA, Weis FR, O'Brien BL, DiPalma JA. Patient-controlled anesthesia for colonoscopy using propofol: results of a pilot study. South Med J 1998; 91: 560-4.

3 Froeblich F, Thorens J, Schwizer W, et al. Sedation and analgesia for colonoscopy: patient tolerance, pain, and cardiorespiratory parameters. Gastrointest Endosc 1997; 45: 1-9.

4 DiPalma JA, Herrera JL, Weis FR, Dark-Mezick DL, Brown RS. Alfentanil for conscious sedation during colonoscopy. South Med J 1995; 88: 630-4.

5 Greilich PE, Virella CD, Rich JM, et al. Remifentanil versus meperidine for monitored anesthesia care: a comparison study in older patients undergoing ambulatory colonoscopy. Anesth Analg 2001; 92: 80-4.

6 Joo HS, Perks WJ, Kataoka MT, Errett L, Pace K, Honey $R J$. A comparison of patient-controlled sedation using either remifentanil or remifentanil-propofol for shock wave lithotripsy. Anesth Analg 2001; 93: 1227-32.

7 Blair JM, Hill DA, Fee JP. Patient-controlled analgesia for labour using remifentanil: a feasibility study. Br J Anaesth 2001; 87: 415-20.

8 Sztark F, Bourdalle-Badie C, Pariente JL, Lamore A, Gardien PL, Erny P. Sédation contrôlée par le patient avec du propofol au cours de la lithotritie extracorporelle. Ann Fr Anesth Réanim 1999; 18: 409-14.

9 Janzen PR, Christys A, Vucevic M. Patient-controlled sedation using propofol in elderly patients in day-case cataract surgery. Br J Anaesth 1999; 82: 635-6.

10 Servin FS, Raeder JC, Merle JC, et al. Remifentanil sedation compared with propofol during regional anaesthesia. Acta Anaesthesiol Scand 2002; 46: 309-15. 